\title{
Aortic valve endocarditis associated with Legionella infection after Mycoplasma pneumonia
}

\author{
PETER LITTRUP, * JAN KYST MADSEN,* KLAUS LIND $\dagger$ \\ From the ${ }^{\star}$ Medical Department $B$, Rigshospitalet and $\uparrow$ Statens Seruminstitut, Copenhagen, Denmark
}

SUMMARY A 38 year old woman with diabetes mellitus and bronchial asthma was admitted to hospital with pneumonia caused by Mycoplasma pneumoniae; she recovered promptly on erythromycin treatment. Six weeks later she presented with aortic valve endocarditis without concurrent lung disease. A concurrent increase in titres of antibody to Legionella bozemanii, L longbeachae, and $L$ jordanis indicated a Legionella infection.

Legionella infection should be considered, even in the absence of pneumonia, in cases of endocarditis where no other cause can be detected.

Although Legionella is known to cause pericarditis and myocarditis, ${ }^{1}$ only one case of endocarditis associated with Legionella infection has been reported. ${ }^{2}$ This patient had undergone mitral and aortic valve replacement with porcine xenografts one month before the infection.

We describe a woman with aortic valve endocarditis who had a concurrent rise in titres of antibodies to Legionella a month after pneumonia caused by Mycoplasma pneumoniae.

\section{Case report}

A 38 year old white woman was admitted on 19 October 1984 after a six day history of fever (up to $40^{\circ} \mathrm{C}$ ), productive cough, right-sided pleuritic chest pain, and dyspnoea on exertion. She had insulin dependent diabetes mellitus and bronchial asthma, but no history of heart disease.

Pneumonia caused by M pneumoniae was diagnosed on the basis of her symptoms, clinical findings, a chest $x$ ray showing infiltration of the right lower and middle lobes, and a significant rise in the titre of complement fixing antibodies to $M$ pneumoniae and a high titre of cold agglutinins ${ }^{3}$ (figure). The heart sounds were normal. Investigations showed: erythrocyte sedimentation rate $120 \mathrm{~mm} / \mathrm{h}$, leucocyte count $10.8 \times 10^{9} / 1(71 \%$ neutrophils, $23 \%$ lymphocytes), blood pressure

Requests for reprints to Dr Jan Kyst Madsen, Medical Department B, Rigshospitalet, University Hospital, Blegdamsvej 9, DK 2100 Copenhagen, Denmark.
$130 / 80 \mathrm{~mm} \mathrm{Hg}$. Blood, throat, and sputum cultures yielded no pathogens. Serological tests for ornithosis were non-diagnostic. The patient was given erythromycin $500 \mathrm{mg}$ three times a day for two weeks and there was a prompt clinical recovery. A chest $x$ ray after discharge (23 November 1984) was normal.

The patient returned to the clinic on 19 December 1984 complaining of general malaise and fever (up to $39^{\circ} \mathrm{C}$ ) at night. On physical examination she was afebrile with a heart rate of 76 beats $/ \mathrm{min}$ and a blood pressure of $170 / 90 \mathrm{~mm} \mathrm{Hg}$. A low frequency, pansystolic, crescendo murmur (grade 3/6) with radiation to the left axilla was heard on auscultation. No diastolic murmur was heard. There was no oedema or cyanosis. Auscultation of the lungs and the rest of the physical examination showed nothing abnormal. On admission investigations showed: haemoglobin $10.6 \mathrm{mmol} / \mathrm{l}$, leucocytes $6.2 \times 10^{9} / 1(40 \%$ neutrophils, $54 \%$ lymphocytes). Chest $x$ ray and electrocardiogram were within normal limits. An echocardiogram three days after admission showed a thickened bicuspid aortic valve with a $5-7 \mathrm{~mm}$ vegetation and a $3 \mathrm{~mm}$ pericardial effusion.

The titre of M pneumoniae antibodies was still high (2000) although the cold agglutinin titre had fallen to 8 (figure). Initial blood cultures for pathogenic bacteria were negative on six occasions and so were cultures from urine, throat, and sputum samples. Cultures from throat (1 January) and blood (18 January) were negative for M pneumoniae. Tests for antibodies to Coxiella burnetii, Chlamydia psittaci, and Brucella abortus were negative. We tested serum samples for antibodies to Legionella, including sera 


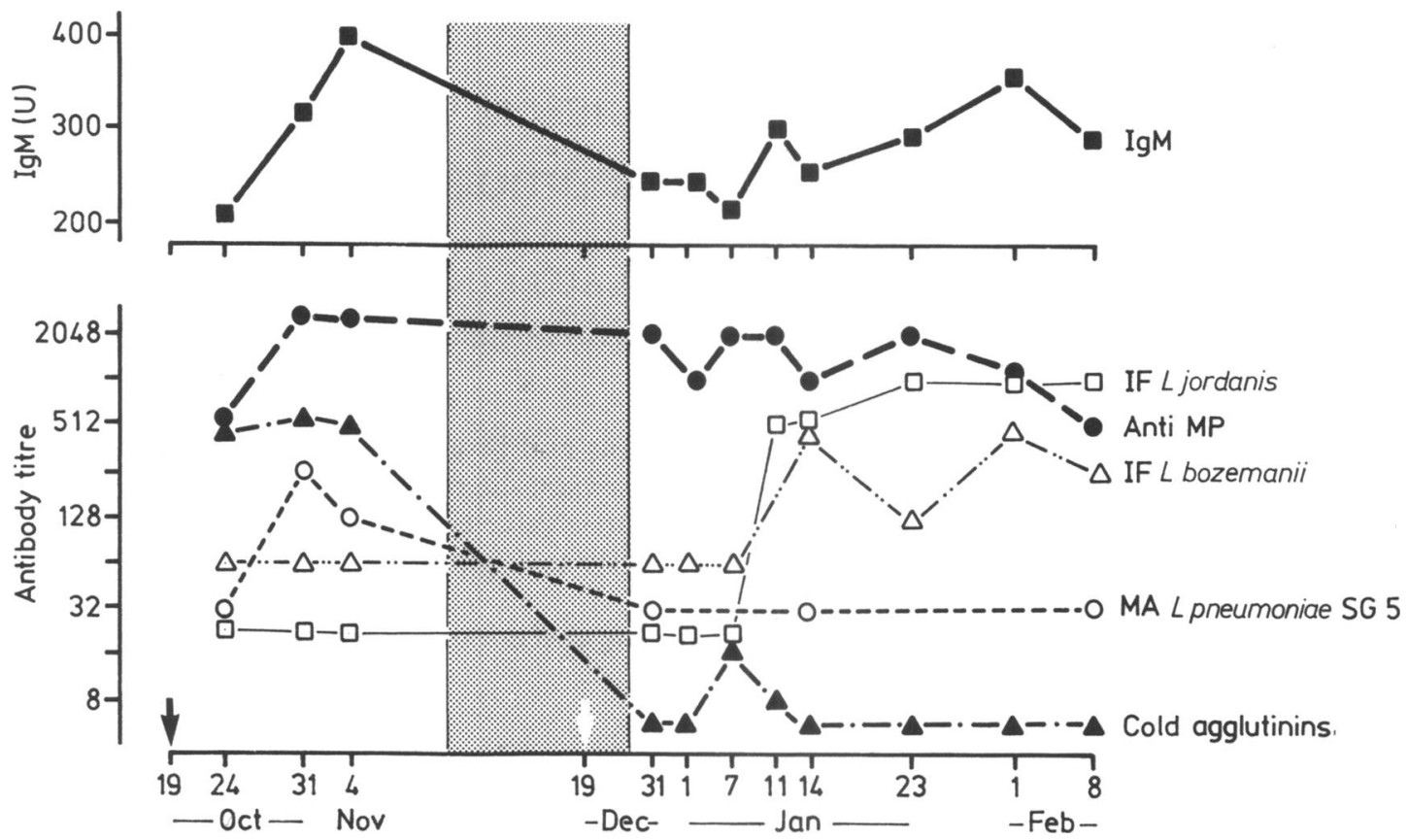

Figure The patient was admitted with pneumonia on 19 October (black arrow) and readmitted with endocarditis on 19 December (white arrow). The diagram shows antibody titres by indirect immunofluorescence (IF) to Legionella jordanis and to Legionella bozemanii; by microagglutinin (MA) to Legionella pneumophila serogroup 5; to anti-Mycoplasma pneumoniae (MP) by complement fixation. Cold agglutinin titres and total serum IgM (normal range 80-322 units) are also shown. The stippled area indicates a period without blood sampling.

from the first admission in October, by the indirect immunofluorescence test and all were negative for the following antigens: $L$ pneumophila, serogroups (SG) 1-6, L micdadei, Lbozemanii, $L$ dumoffi, L gormanii, L longbeachae, SG 1 and 2, and $L$ jordanis. ${ }^{4}$

The patient continued to have fever $\left(38.5^{\circ} \mathrm{C}-39.0^{\circ} \mathrm{C}\right)$ at night. Twelve further blood specimens were drawn at 11 and $12 \mathrm{pm}$ and $1 \mathrm{am}$ on four consecutive nights; they remained negative on culture. A repeat echocardiogram on 2 January 1985 showed no change in the aortic valve vegetations, but mild, apparently haemodynamically insignificant aortic regurgitation was noted. Empiric antibiotic treatment with intraveneous penicillin $5 \mathrm{M}$ IU four times a day and netilmicin $100 \mathrm{mg}$ three times a day was started on 3 January. Nightly fever spikes continued, and titres of antibodies to L bozemanii and L longbeachae SG 1 and 2 rose significantly to 512 while antibody titres to L jordanis rose to 1024 in the indirect immunofluorescence test in which "blocking fluid" extracted from Escherichia coli was used as a serum diluent $^{5}$ (figure). Mycoplasma antibody titres remained at 1000-2000. Antibiotic treatment was switched to intravenous erythromycin $1 \mathrm{~g}$ four times a day from 18 January, and after three days the nightly fever spikes abated.

Chest $x$ ray was still normal and an echocardiogram on 18 February was unchanged. After 6 weeks' treatment, intravenous erythromycin was changed to $1 \mathrm{~g}$ orally three times a day; this treatment was given for two weeks.

\section{Discussion}

This patient presented with two episodes of infectious disease. The first was severe acute pneumonia which was believed to be caused by $M$ pneumoniae because there was a rise in specific antibody titre and a high transitory cold agglutinin titre. Attempts to isolate the organism two months after the acute illness were unsuccessful. One month after presenting with pneumonia the patient had an aortic valve endocarditis confirmed by clinical findings and echocardiography. The endocarditis was accompanied by a considerable rise in titre of antibodies to Legionella, indicating a causal assocation; however, we did not attempt to isolate the organism and there were no signs of pulmonary infection. 
We considered the possibility that during the first episode $M$ pneumoniae might have evoked an anamnestic response of antibodies to Legionella because M pneumoniae is a known to be a polyclonal B-cell activator. ${ }^{6}$ Therefore, concentrations of total serum IgM and IgG were measured retrospectively in all serum specimens. ${ }^{7}$ The results (figure) indicate that the typical increase in serum IgM during the $M$ pneumoniae infection had abated before the endocarditis occurred and before the start of the rise in Legionella antibody titre detected by the indirect immunofluorescence test. During the first three weeks of this episode there was a second but less steep increase in serum IgM that was accompanied by a slight drop in Mpneumoniae antibody titre. Changes in IgG concentration followed the IgM changes but were less pronounced (not shown). We also tested all serum specimens for Legionella antibodies in a microagglutination test. ${ }^{8}$ We found a significant rise in titre of antibodies to $L$ pneumophila SG 1 (to 64, not shown) and SG 5 (to 256, figure) during the first episode that paralleled the rise in total IgM. Antibodies to the other 11 Legionella antigens mentioned above remained at the same titre until the episode of endocarditis, when titres of antibodies to L bozemanii and L longbeachae SG 1 and 2 also rose in the microagglutination test (not shown in figure).

We believe that the first rise in antibodies to $L$ pneumophila serogroup 1 and 5 was an anamnestic response and that the later increase in titre of both immunofluorescent and microagglutination antibodies to Lbozemanii and Llongbeachae indicates that the endocarditis may have been caused by a Legionella infection. The patient had insulin dependent diabetes and a chronic bronchial asthma; both increase the risk of Legionella infection. This risk was probably further increased by the previous $M$ pneumoniae infection, which is known to depress cellular immunity. ${ }^{9}$

In the other report on a case of endocarditis caused by $L$ pneumophila, the heart was infected in the absence of pneumonia ${ }^{2}$; this was the case in our patient too. The species or serogroup of Legionella which may have caused the infection in our patient cannot be deduced from antibody determination because of interspecies, often unpredictable, antigenic cross-reactions. ${ }^{5}$ When antibody titres to other pathogens increase during an infection with
M pneumoniae serodiagnosis is difficult because this organism is known to cause anamnestic antibody responses. ${ }^{3}$ Several studies have found that antigens of $M$ pneumoniae do not cross react serologically with Legionella antigens. ${ }^{5}$

We conclude that the possibility of Legionella infection should be considered in cases of endocarditis where no other cause can be shown, and that the absence of pneumonia does not exclude the possibility of a Legionella infection.

We thank Bendt Mansa, Deparment of Biophysics, Statens Seruminstitut, for measuring serum IgM and IgG.

\section{References}

1 Woodhead MA, MacFarlane JT. The protean manifestations of Legionnaires' Disease. J $R$ Coll Physicians Lond 1985;19:224-30.

2 McCabe RE, Baldwin JC, McGregor CA, Miller DC, Vosti KL. Prosthetic valve endocarditis caused by Legionella pneumophila. Ann Intern Med 1984; 100:525-7.

3 Lind K, Bentzon MW. Epidemics of Mycoplasma pneumoniae infection in Denmark from 1958 to 1974. Int $J$ Epidemiol 1976;5:267-77.

4 Lind K. Serological investigations of Legionella infections in Denmark. Acta Pathol Microbiol Immunol Scand [B] 1983;91:209-13.

5 Wilkinson HW, Reingold AL, Brake BJ, McGiboney DL, Gorman GW, Broome CV. Reactivity of serum from patients with suspected legionellosis against 29 antigens of legionellaceae and Legionella-like organisms by indirect immunofluorescence assay. $J$ Infect Dis 1983;147:23-31.

6 Biberfeld G, Arneborn P, Forsgren M, von Stedingk LV. Non-specific polyclonal antibody response induced by Mycoplasma pneumoniae in vitro and in vivo. Yale J Biol Med 1984;57:893.

7 Vejtorp M, Mansa B. Rubella IgM antibodies in sera from infants born after maternal rubella later than the 12th week of pregnancy. Scand J Infect Dis 1980;12:1-5.

8 Lind K, Collins MT, Aalund O. Comparison of a microagglutination test and the indirect immunofluorescence test for Legionella antibodies in patients. Acta Pathol Microbiol Immunol Scand [B] 1984;92: 195-9.

9 Biberfeld G, Sterner G. Effect of Mycoplasma pneumoniae infection on cell-mediated immunity. Infection 1976;4(suppl 1):17-20. 